\title{
The Incidence Trends of Primary Brain Tumors in Saskatchewan from 1970 to 2001
}

\author{
F. Pirouzmand, V. Sadanand
}

\begin{abstract}
Objective: There has been a paucity of information on the epidemiology of primary brain tumors (BTs) in Canada. This study documents epidemiology of primary BTs in Saskatchewan over three decades to define their current state, changing pattern over years and relative distribution in two geographically defined areas of the province. Methods: Data on all primary BTs from 1970 to 2001 from the Brain Tumor Registry in Saskatchewan was collected. The aggregate data on primary BTs including the time-series for incidence, age, geographic location and sex were statistically analyzed using SPSS 13. Poisson regression was used to model the incidence as a function of decade of diagnosis and age at the time of diagnosis. Results: The average annual incidence of primary BTs was 11.1 per 100,000 person-years (male 12.5 per 100.000 and female 9.8 per 100.000). Males constituted $54.5 \%$ of all these tumors. The age distribution of tumors was bimodal with peaks at 5 years and 65 years. During this time, the incidence of primary BTs has increase predominantly in non-malignant types. No difference was found in the rate of all the diagnosed primary BTs combined, meningioma and lymphoma between the northern part (Regina) and southern part (Saskatoon) of the province. Conclusions: The incidence of BTs in Saskatchewan is more than previously reported in Canada. There is a temporal trend in increasing incidence of some of the BTs predominantly in the non-malignant types. No spatial difference in the incidence of primary BTs was shown in this province. These data will provide useful information to guide the future studies on BTs changing patterns, possible etiologies and efficient resource allocation for management of these diseases.
\end{abstract}

RÉSUMÉ: Évolution de l'incidence des tumeurs primitives du cerveau en Saskatchewan de 1970 à 2001 . Objectif : Il y a peu d'information sur l'épidémiologie des tumeurs primitives du cerveau (TCs) au Canada. Cette étude fait état de l'épidémiologie des TCs primitives en Saskatchewan pendant trois décennies afin de décrire la situation actuelle, les changements dans le temps et la distribution relative dans deux régions de la province qui sont bien définies au point de vue géographique. Méthodes : Nous avons extrait les données contenues dans le registre des tumeurs cérébrales de la Saskatchewan concernant toutes les TCs primaires de 1970 à 2001. Nous avons analysé l'agrégat de données sur les TCs primaires, dont les séries temporelles pour l'incidence, l'âge, le lieu géographique et le sexe au moyen du SPSS 13. Nous avons utilisé la régression de Poisson pour modéliser l'incidence comme fonction de la décade du diagnostic et de l'âge au moment du diagnostic. Résultats : L'incidence annuelle moyenne des TCs était de 11,1 par 100000 personnes-années (12,5 par 100000 pour les hommes et 9,8 par 100000 pour les femmes). 54\% des patients étaient des hommes. La distribution de l'âge était bimodale, avec un pic à 5 ans et un pic à 65 ans. Pendant la période étudiée, l'incidence des TCs a augmenté surtout en ce qui concerne les tumeurs bénignes. Il n’y avait pas de différence dans le taux de toutes les TCs combinées, le méningiome et le lymphome entre la partie nord (Regina) et la partie sud (Saskatoon) de la province. Conclusions : L'incidence des TCs en Saskatchewan est plus élevée que celle rapportée antérieurement au Canada. On observe une tendance temporelle à l'augmentation de l'incidence de certaines TCs, surtout des tumeurs bénignes. Aucune différence n'a été observée dans l'incidence des TCs primitives entre les régions de cette province. Ces données pourront servir de guide dans l'élaboration d'études éventuelles sur l'incidence, les étiologies possibles et l'allocation des ressources pour le traitement de ces maladies.

Can. J. Neurol. Sci. 2007; 34: 181-186

The incidence of brain tumors may vary in different geographic locations and over time. There has been little information on the epidemiology of brain tumours (BTs) in Canada. ${ }^{1}$ With soaring health care costs; the emphasis on resource allocation requires an understanding of the epidemiology of health care. In the province of Saskatchewan there have been important changes over the last 30 years. Population growth, lifestyle progress, agricultural and technological improvements, mining and manufacturing developments have all had a cumulative impact on individual lives. The impact of these changes on the health of its population is less well understood. In this paper, we explore a 32 year long-

From the Division of Neurosurgery, Sunnybrook Health Sciences Centre (FP), University of Toronto, Toronto, ON; Division of Neurosurgery, Royal University Hospital (VS), Saskatoon, SK, Canada.

Received November 30, 2004. Accepted in final form December 18, 2006. Reprint requests to: F. Pirouzmand, Division of Neurosurgery, Sunnybrook Health Sciences Centre, University of Toronto, Suite A131, 2075 Bayview Ave., Toronto, Ontario, M4N 3M5, Canada. 
term data on 2468 patients from 1970-2001 with primary BTs. The data was collected from the Central Brain Tumor Registry of Saskatchewan which is the comprehensive data bank for all the BTs including metastatic tumors in the province. Only primary BT data was extracted for the purpose of this study.

Although this is the first large scale long term populationbased study of BTs in this province, there have been other studies conducted in Canada and in the world. Of note in these studies is the overall incidence rate of primary BTs ranging from 6.1 per 100,000 person per year in a recent American study based on three decades of data; ${ }^{2} 10.6$ to 15.5 in a French study. ${ }^{3}$

As correctly pointed out previously, ${ }^{1}$ the geographical scope of our data minimizes selection bias inherent in hospital based studies. In addition, the province of Saskatchewan has had a somewhat stable demography (population and the age distribution) during these three decades and the only referral centers for BTs have remained the same during this period. Our goal is to understand the incidence trend of such tumors to enable us to efficiently allocate resources for the diagnosis, screening, possible prevention and management of these patients. Better understanding of distribution of these tumors both temporally and spatially could also theoretically provide information on possible etiological factors.,

\section{Materials ANd Methods}

\section{Source of data}

Data was collected on all primary BTs from 1970 to 2001 from the Brain Tumor Registry in Saskatchewan. This registry covers all the diagnosed brain cancer patients in the province of Saskatchewan in this period. In this study, the metastatic diseases were excluded from the collected data for final analysis. The Saskatchewan Cancer Commission Act of 1930 provided authorization for the establishment of a cancer control program in Saskatchewan. In 1932, comprehensive diagnostic and treatment services for cancer for all residents of the province were initiated. A population-based registration dates from this time. The registry is respected for its meticulous record keeping with tabulated clinico-pathological information. The province is covered by a universal comprehensive health insurance plan. To be eligible for cancer payments under this plan, the physicians are required to report all new cancer diagnoses to the Registry. To complement this, copies of all malignant pathology reports are sent to the appropriate cancer clinic. Further cases are discovered through death certificate notifications which are received on a bi-weekly basis from the Vital Statistics Branch of Saskatchewan Health.

The published census data from statistics Canada in 1971, 1986 and 1996 were used for calculation of incidence in the appropriate categories. The incidence was calculated separately for three decades based on the population data in the index year for that decade (i.e. 1971 for 70s decade, 1986 for 80s decade and 1996 for 90 s decade). The population was divided into three groups as follow: young age 0-19, adult age 20-65 and old age $65<$. The age group incidence was calculated accordingly for each category over three decades.

\section{Brain tumor classification}

The incident cases diagnosed from 1970 through 1976 were classified according to the Manual of Tumor Nomenclature and
Coding. ${ }^{6}$ The tumors diagnosed from 1977 through 1991 were classified according to the International Classification of Diseases for Oncology (ICD-O) ${ }^{7}$ and those diagnosed from 1992 onward were classified according to International Classification of Diseases for Oncology, 2nd edition (ICD-O-2). ${ }^{8}$

We included all histological diagnosis in the study. There were no exclusion criteria. The tumors were classified into "malignant" and "non-malignant" forms based on the following. All tumors with extension diagnostic code of 3 were labeled as malignant and tumors with extension code 0 (i.e. benign) and extension code 1 (i.e. uncertain whether benign or malignant) were classified as "non-malignant".

The proportion of cases with microscopically proven pathology was recorded for each year.

The aggregate data on all the primary BTs including the sex, and age distribution was collected. The patients were divided into three age group category $(<20,20-65,65<)$ to calculate age specific incidence. To explore any changing pattern in the type of pathology of tumors, the incidence of malignant and nonmalignant tumors in each age group was calculate over time period of study.

The province of Saskatchewan has only two neurosurgical centers: Saskatoon and Regina centers. It was assumed that the data obtained from the Saskatoon cancer center represented patients that were domicile in the northern half of the province while the data obtained from the Regina cancer center represented patients domicile in the southern half of the province. This is a reasonable assumption given that both centers had state-of-the-art cancer treatment facilities and cover the specified catchments area. Historically, the Saskatoon center has been covering about $60 \%$ of the population (based on the catchments area) and the Regina center responsible for the rest.

The overall frequency of all the primary BTs combined was statistically compared between these two geographically distinct parts of the province (i.e. Saskatoon representing the upper part and Regina the lower part). This overall frequency was also calculated separately for meningioma and lymphoma between these two regions. These tumors were selected as representative of "non-glial" primary BTs.

\section{Statistical analysis}

Incidence rate was calculated per 100,000 person-years. The age at diagnosis is the known factor affecting brain tumor characterization. Therefore, age-specific incidence for three defined age groups (see above) was also calculated. Demographic data on age, sex and incidence was analyzed using univariate techniques. The difference in the ratio for various tumors diagnosed between the two parts of the province was also assessed with simple ratio comparison. Numerous prognostic factors are potentially associated with the incidence trend over time. The effect of age group and decade of diagnosis was assessed in this study. We used log-linear model assuming a Poisson distribution to model the incidence rate as a function of decade of diagnosis and age group for all brain tumors combined, only non-malignant and only malignant brain tumors. The histological confirmation for each case and the overall percentage of confirmed tissue diagnosis was also recorded. The SPSS 13.0 for Windows software (SPSS Inc. Chicago, IL. 2004) was used to calculate all the results. We used a type I error rate of $5 \%$ to determine statistical significance. 
Table 1: Characteristics of primary brain tumors in 2468 patients in Saskatchewan from 1970-2001

\begin{tabular}{|c|c|c|c|c|}
\hline Histology type & No. of cases $(\%)$ & Median age (Yrs) & Male (\%) & Female $(\%)$ \\
\hline Glioma, NOS (9380) & 124 & 53 & $70(56 \%)$ & $54(44 \%)$ \\
\hline GBM (9440) & 531 & 64 & $321(60 \%)$ & $210(40 \%)$ \\
\hline Astrocytoma, NOS & 578 & 54 & $352(61 \%)$ & $226(39 \%)$ \\
\hline Oligodendroglioma, NOS (9450) & 80 & 44 & $52(65 \%)$ & $28(35 \%)$ \\
\hline \multicolumn{5}{|c|}{ Oligodendroglioma, anaplastic (9451) } \\
\hline Pituitary tumors & 171 & 52 & $91(53 \%)$ & $80(47 \%)$ \\
\hline Lymphoma & 60 & 64 & $26(43 \%)$ & $34(57 \%)$ \\
\hline Meningioma & 319 & 62 & $120(36 \%)$ & $209(64 \%)$ \\
\hline Anaplastic Astrocytoma (9401) & 94 & 53 & $50(53 \%)$ & $44(47 \%)$ \\
\hline \multicolumn{5}{|l|}{ Protoplasmic Astrocytoma (9411) } \\
\hline \multicolumn{5}{|l|}{ Gemistocytic Astrocytom (9411) } \\
\hline \multicolumn{5}{|l|}{ Fibrillary Astrocytoma (9420) } \\
\hline All other tumors combined & 511 & 47 & $266(52 \%)$ & $235(48 \%)$ \\
\hline
\end{tabular}

\section{RESULTS}

\section{Demographics}

Table 1 summarizes the distribution of tumor type, sex and age for 2468 primary intracranial tumors during the study period. The average annual incidence of brain tumors was 11.1 per 100,000 person-years (male 12.5 per 100.000 and female 9.8 per 100.000). Males constituted $54.5 \%$ of all these tumors.

\section{Brain cancer trends (temporal)}

The age distribution of tumors was bimodal with peaks at early childhood (5 years) and more pronounced peak in elderly (65 years). This is shown in Figure 1. The age-group specific incidence rate over three decades of study for all non-malignant and malignant brain tumors are depicted in Figure 2. This reveals an overall raise in the incidence of brain tumors with increasing age group and over the time span of study. This is shown in Figure $3 \mathrm{a}$ for all brain tumors combined. The majority of this increase is accounted for by significant increase in nonmalignant brain tumors (Figure 3b). The curve for the time period effect in malignant brain tumors remains relatively flat (Figure 3c).

The results of period incidence from Poisson regression model are shown in Table 2. There is significant increase in the incidence of all brain tumor combined and benign tumors over the study period and for each increasing age group category $(\mathrm{P}<0.05)$. This model also reveals interaction between the age at the diagnosis (i.e. second group) and year of diagnosis (i.e. first decade of study) only for all brain tumors analysis (not shown in table). In contrast, for malignant brain tumors only the age at the diagnosis is significant. There has been no significant change over the last two decades of study in incidence of malignant brain tumors.

\section{Brain cancer trends (spatial)}

From a geographical point of view within the province, the ratio of all the diagnosed brain tumors between Saskatoon and
Regina was 1.4 (1272/1117 tumors). The similar ratio for the meningioma and lymphoma was $1.2(130 / 108)$ and $1.4(35 / 26)$ respectively. These ratios are not significantly different and correspond to the catchments area for the two centers and relative workload.

\section{Histological confirmation trends}

The proportion of cases confirmed microscopically was on average $45 \%$ before 1980 and $86 \%$ after 1980 . This highlights a

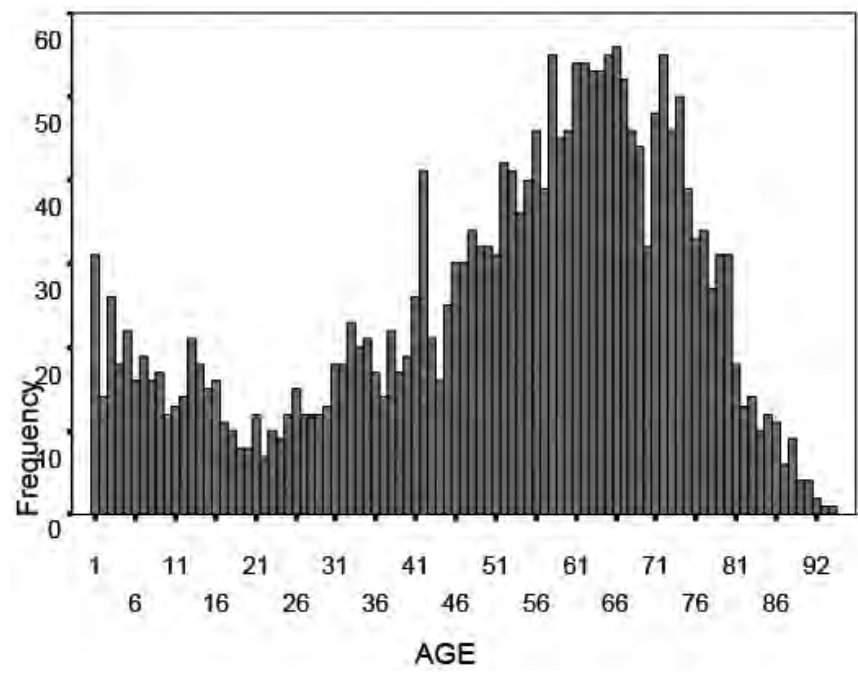

Figure 1: This bar diagram shows the cumulative frequency of all primary BTs in different ages during the three decades of study. A standard bimodal distribution is seen. This suggests possibly two overlapping distributions for the pediatric and adult groups rather than measurement error. 


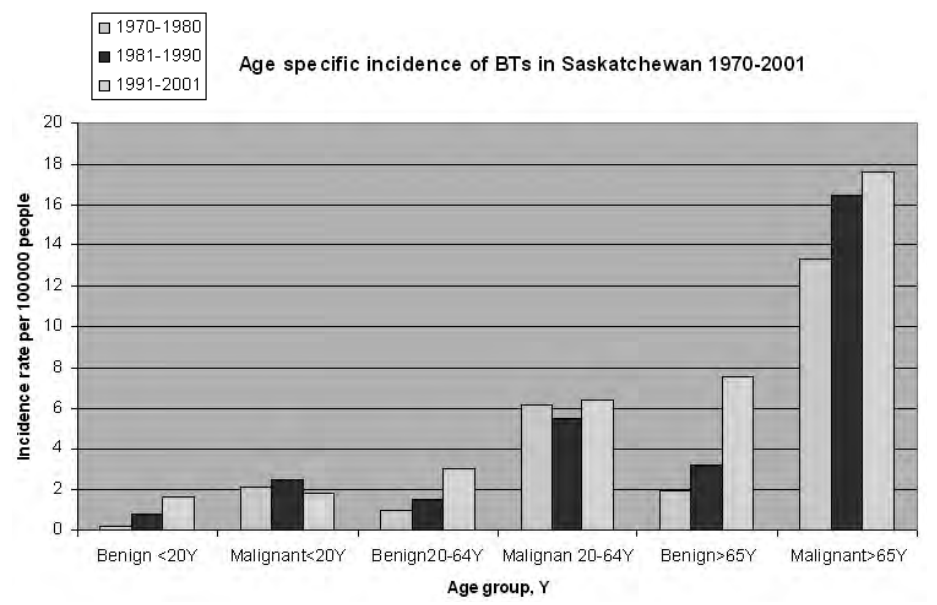

Figure 2: The age-group specific incidence rate over 3 decade of study for all primary benign and malignant brain tumors is shown. Note the overall increase in the incidence with higher age group. significant shift toward more aggressive histological confirmation in diagnosis and subsequent management of these patients as a group.

\section{Discussion}

In this study, we determined the incidence of all primary brain tumors as 11.1 per 100,000 person per year. According to previously published data the incidence varies from 6.1 to 15.5 cases per 100,000 person per year. ${ }^{1-3,9-13}$ Our result is higher than SEER (Surveillance, Epidemiology, and End Results) registry result of 6.1 published in 2006..$^{2,14}$ In this report, the authors reviewed the data on primary brain tumors in SEER database from 1973 to 2001. They also found that the incidence of brain cancer increased until 1987, when the annual percentage of change reversed direction, decreasing from +1.68 to $-0.44 \%$. $^{2}$

A thorough analysis of the epidemiology of primary intracranial neoplasms in Manitoba was conducted by Sutherland et al in 1987. ${ }^{1}$ The incidence was reported as 10.1 in this 6-year study from 1980-1985. The authors not only accessed brain tumor registry data, but also reviewed

Age group incidence rate of all BTs in Saskatche wan 1970-2001
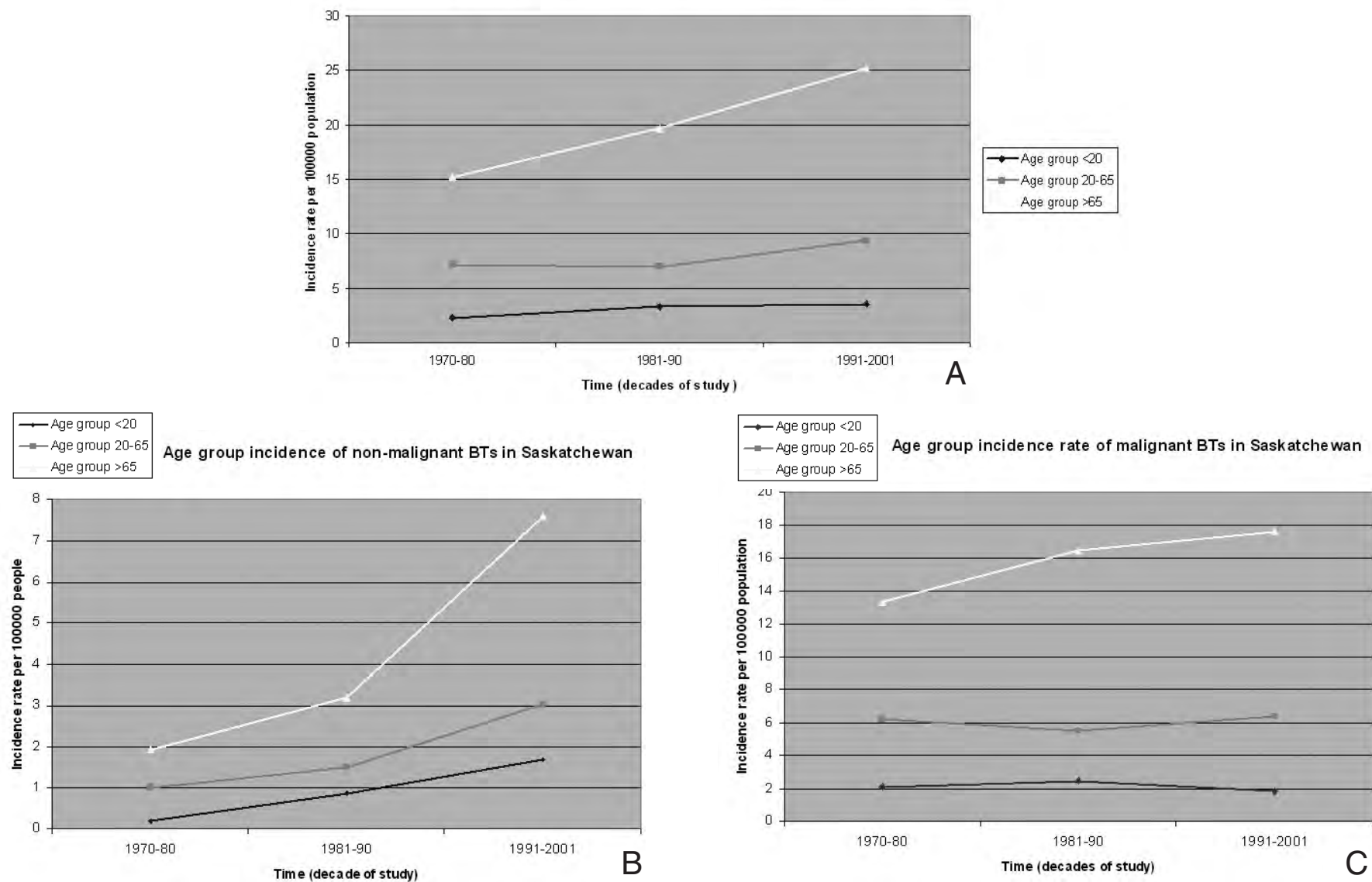

Figure 3: The incidence trend over study period is shown for all primary brain tumors combined (3a), non-malignant tumors (3b) and malignant brain tumors $(3 \mathrm{c})$. Note that the curvature line is relatively flat for malignant tumors in all age groups in contrast to steadily raising trend in non-malignant tumors. 
Table 2: Relative risk of developing primary brain tumor in the province of Saskatchewan over 3 decades (Results are adjusted for age groups and decade of cancer registration using Poisson analysis)

\begin{tabular}{|c|c|c|c|c|c|c|}
\hline \multirow[t]{2}{*}{ Independent variable } & \multicolumn{6}{|c|}{ Relative Risk (RR) } \\
\hline & All BTs & Sig. & $\begin{array}{c}\text { Benign } \\
\text { BTs }\end{array}$ & Sig. & $\begin{array}{c}\text { Malignant } \\
\text { BTs }\end{array}$ & Sig \\
\hline Age group $\mathrm{I}<20 \mathrm{Y}$ & 0.1 & .00 & 0.2 & .00 & 0.1 & .00 \\
\hline Age group II 20-64Y & 0.4 & .00 & 0.4 & .00 & 0.4 & .00 \\
\hline Age group III >65Y & 1.00 & - & 1.0 & - & 1.0 & - \\
\hline 1st decade $1970-1980$ & 0.6 & .00 & 0.3 & .00 & 0.8 & .00 \\
\hline 2nd decade 1981-1990 & 0.7 & .00 & 0.4 & .00 & 0.9 & - \\
\hline 3rd decade 1991-2001 & 1.0 & - & 1.0 & - & 1.0 & - \\
\hline
\end{tabular}

clinical, radiological, surgical and histopathological correlates. This was especially relevant in their study for tumor types where the disease incidence was higher than previously reported. Our report differs from this approach. Our data spans 32 years and 2468 patients. Some of our data is from a period when prevailing radiological technology would be considered inadequate by today's standards. We didn't have access to additional clinical, surgical and imaging information other than that provided by the detailed record-keeping by the brain tumor registry.

Some of the above differences in reported incidence of primary brain tumors are presumably a reflection of the type of medical care, availability of diagnostic tools and reliability of the registries for data collection and coding procedure. More accessible medical care, improved socioeconomical status and more aggressive diagnostic investigation (e.g. CT or MR) have certainly contributed to the increased rate of BT diagnosis. In Annegres et al. paper the incidence rate in Rochester, Minnesota was $14.5 \% .^{9}$ This may in part be due to better diagnosis and post mortem reporting included in the final analysis.

The current study reveals a significant increase in the incidence over the study period in all age groups. With regard to histological types, it shows a significant difference in the rate of incidence change for non-malignant and malignant brain tumors over the study period. This somewhat surprising result is not consistent with other published data. One possible explanation is a better chance for diagnosis of non-malignant brain tumors (which could be relatively asymptomatic) with ongoing advancement in technology. Another important conclusion is lack of significant change in the incidence of relatively uniform group of tumors labeled as malignant in this study.

There was no significant difference between the two geographic parts of the province in the rate of diagnosis for all the BTs combined or for selected types of tumors in this study (i.e. meningioma or lymphoma). Note that due to the artificial division of the province, accurate calculation of incidence was not possible. The ratio for the diagnosis (i.e. 1.2-1.4) of these tumors was compatible with the original assumption of relative patient loads of Saskatoon and Regina centers (i.e. 60\%/40\%). However, the geographic differences may still be present but not to the degree to allow demonstrable differences (i.e. low power of study).
The present study is a retrospective time series data analysis and such studies have well-known disadvantages. First, data collection techniques may change over time. For example, over the 33 years, different tumor grading systems have evolved. This renders the pathological classification somewhat non-uniform throughout the study time line. Furthermore, changes in diagnostic and operative techniques have resulted in increasingly more operative interventions, hence increasing volume of surgical pathology. This is clearly shown by the increase in pathological confirmation over study time in the result (from $45 \%$ to $86 \%$ before and after 1980). Second, longitudinal studies have posed difficulties with viewing fluctuations in more than one variable at a time. Poisson models could overcome some of these limitations. Third, the results are not meant to suggest that current trends will continue. However, they provide an insight into possible independent variables that may explain the tumor trends. Finally, longitudinal studies have statistical biases such as selection, treatment, measurement and aggregation biases that weaken the internal validity of the results. These are unavoidable and must be considered in assessing the overall import of the results.

It is important to note that the division of all primary tumors into malignant and non-malignant is based on somewhat arbitrary definition of codes (see Methods section). In each group there is a wide range of potentially diverse and unique pathologies grouped under one umbrella. Therefore the changing patterns of malignant and non-malignant tumors is not tissue specific but provides a general trend of information on specifically defined group of tumors. The current asymmetric increase in non-malignant versus malignant primary BTs need further epidemiological study to decipher if for example this is related to a more specific type of tumor.

The analysis of a large time-series data on primary BTs tumors in Saskatchewan over a period of three decades has shown slightly higher than expected incidence of BTs. The changing temporal pattern of some BTs (i.e. increasing nonmalignant brain tumors) and lack of spatial differences between northern and southern part of province requires further research to examine the etiological and explanatory factors. This data will provide useful information to guide the future studies on BT 
changing patterns, possible etiologies and efficient resource allocation for the management of these diseases.

\section{REFERENCES}

1. Sutherland GR, Florell R, Louw D, Choi NW, Sima AA. Epidemiology of primary intracranial neoplasms in Manitoba, Canada. Can J Neurol Sci. 1987;14:586-92.

2. Deorah S, Lynch CF, Sibenaller ZA, Ryken TC. Trends in brain cancer incidence and survival in the United States, SEER program, 1973 to 2001. Neurosurg Focus. 2006;20:1-7.

3. Elia-Pasquet S, Provost D, Jaffre A, Loiseau H, Vital A, Kantor G, et al. Incidence of central nervous system tumors in Gironde, France. Neuroepidemiology. 2004;23:110-7.

4. McKinney PA. Brain tumours: incidence, survival, and aetiology. J Neurol Neurosurg Psychiatry. 2004;75 Suppl 2:ii12-ii17.

5. Wrensch M, Minn Y, Chew T, Bondy M, Berger MS. Epidemiology of primary brain tumors: current concepts and review of the literature. Neuro Oncol. 2002;4:278-99.

6. American Cancer Society. Manual of tumor nomenclature. New York: American Cancer Society, 1951.

7. World Health Organization. International classification of diseases for oncology. Geneva: World Health Organization; 1976.
8. World Health Organization. International classification of diseases for oncology. Geneva: World Health Organization; 1990.

9. Annegers JF, Schoenberg BS, Okazaki H, Kurland LT. Epidemiologic study of primary intracranial neoplasms. Arch Neurol. 1981;38:217-9.

10. Hess KR, Broglio KR, Bondy ML. Adult glioma incidence trends in the United States, 1977-2000. Cancer. 2004;101:2293-9.

11. Johannesen TB, Angell-Andersen E, Tretli S, Langmark F, Lote K. Trends in incidence of brain and central nervous system tumors in Norway, 1970-1999. Neuroepidemiology. 2004;23:101-9.

12. Jukich PJ, McCarthy BJ, Surawicz TS, Freels S, Davis FG. Trends in incidence of primary brain tumors in the United States, 19851994. Neuro Oncol. 2001;3:141-51.

13. Kuratsu J, Ushio Y. Epidemiological study of primary intracranial tumors: a regional survey in Kumamoto Prefecture in the southern part of Japan. J Neurosurg. 1996;84:946-50.

14. Surveillance Epidemiology, and End Results (SEER) program: National Cancer Institute. 2006. Ref Type: Internet Communication. Available at: http://seer.cancer.gov/ 